ARTÍCULO ORIGINAL

\title{
Intervención del ser humano en el proceso de destete de cabritos lecheros: parámetros fisiológicos y comportamiento animal
}

\author{
Martínez $\mathrm{GM}^{1 *}$, Pereyra $\mathrm{WR}^{2}$, Suarez $\mathrm{VH}^{2}$, Alfaro $\mathrm{EJ}^{1}$, Alfaro $\mathrm{RJ}^{1}$, Yanez $\mathrm{RP}^{1}$ \\ ${ }^{1}$ Instituto Nacional de Tecnología Agropecuaria (INTA) EEA Salta, Argentina. \\ 2 INTA Área de Inv. Salud Animal -IIACS con sede en EEA Salta, Argentina. \\ * Correspondencia: Gabriela Martinez. INTA EEA Salta. Ruta Nacional 68 km 172 \\ (CP: 4403) Cerrillos, Salta, Argentina. \\ E-mail: martínez.gabriela@inta.gob.ar
}

Recibido: 4 Septiembre 2018. Aceptado: 17 Octubre 2018. Disponible en línea: 30 Octubre 2018

Editor: R. Cerutti

RESUMEN. La intervención de los seres humanos en los procesos de cría y recría en animales de producción pueden tener diferente impacto en virtud al tipo, momento y duración de la misma. El objetivo del trabajo fue evaluar si existen cambios en parámetros fisiológicos, sanguíneos y de comportamiento en cabritos Saanen en función al tipo de crianza, 10 criados con sus madres (CT) vs. 10 criados artificialmente (CA), en las etapas de lactante y recría. Semanalmente se midió: frecuencia cardiaca ( $F C$ ), temperatura rectal (TR), frecuencia respiratoria (FR), predisposición a ser atrapado y actitud de patear. Previo se tomaron $10 \mathrm{ml}$ de sangre para realización del leucograma. El diseño experimental fue el de mediciones repetidas en el tiempo. Los datos fueron analizados a través del programa InfoStat. Ni entre periodos ni entre tratamientos se detectaron diferencias significativas para FC como TR. En ambos periodos a los animales del CT resultó más difícil atraparlos (lactante: $p<0,0001$, recría: $p<0,0020$ ). En la etapa de lactantes la cantidad de animales que pateaban fue superior $(p<0,05)$ en $C T$, mientras que en recría no existieron diferencias. Durante la etapa de lactantes los conteos de neutrófilos del grupo CT fueron mayores $(p<0,0001)$ y los de linfocitos menores $(p<0,0001)$. Durante recría se hallaron diferencias $(p<0,0001)$ entre grupos y la relación neutrófilos/linfocitos fue superior $(p<0,0001)$ en un $50 \%$ en los CT. En función a lo obtenido es posible concluir que si bien existe un grado de estrés cuando el animal no habituado al ser humano se enfrenta a éste en manejos que ameriten su captura, el contacto en etapas tempranas permite aumentar el nivel de docilidad a la vez facilita la ejecución de tareas rutinarias.

SUMMARY. Human intervention in dairy kids weaning process: physiological parameters and animal behavior. Human intervention in breeding and rearing processes may have different impact in animals related to the type, moment and duration. The aim of the study was to evaluate if there are changes in physiological, blood and behavioral parameters in Saanen kids according to type of type of breeding, 10 raised with their mothers (CT) vs. 10 artificially reared (CA), in pre-weaning and post-weaning stages. Heart rate (HR), rectal temperature (TR), respiratory frequency (FR), predisposition to be caught and kicking attitude were measure once a week. Prevously, $10 \mathrm{ml}$ of blood was collected for leukogram determination. Experimental design was repeated measurements in CRD. Data were analyzed by InfoStat program. Neither between periods nor between treatments were significant differences detected for HR as TR. CT animals were more difficult to catch in both periods (pre-weaning: $p<0.0001$, post-weaning: $p<0.0020$ ). In pre-weaning stage the number of animals that kicked was higher $(p<0.05)$ in $C T$, whereas during rearing period there were no differences. During pre-weaning, neutrophil counts of the CT group were higher $(p<0.0001)$ and lymphocytes lower $(p<0.0001)$ than in rearing stage. During rearing differences were found $(p<0.0001)$ between groups and the neutrophil / lymphocyte ratio was higher ( $p<0.0001)$ by $50 \%$ in the TC. It is possible to conclude that although animals can become stressed when caught; contact with humans in early stages allows increasing the level of docility and, at the same time, facility the execution of routine tasks.

Palabras clave: tipo de crianza, respuesta conductual, parámetros fisiológicos, leucograma, caprinos

Key words: type of breeding, behavioral response, physiological parameters, leukogram, goats

\section{Introducción}

En las especies domésticas, existen grandes diferencias individuales entre los animales en sus relaciones con el hombre. En los sistemas de producción intensivos el con- tacto de los animales con el ser humano en general es de una frecuencia diaria y se encuentra asociado principalmente a procesos relativos a la alimentación y al cuidado (Miranda-de la Lama y Matiiello, 2010). La interacción humano-animal está condicionada por los 5 sen- 
tidos: tacto, olfato, gusto, audición y visión; y en función a su naturaleza puede ser considerada como positiva, neutra o negativa (Zulkifli, 2013).

Si bien existen patrones de comportamiento entre los animales de la misma especie, varios autores mencionan que el desempeño de los seres humanos que se encuentran al cuidado de los animales tiene gran impacto y puede afectar el bienestar, el comportamiento y la producción (Breuer et al., 2000; Waiblinger et al., 2006; Hanna et al., 2009, Martínez et al, 2016). A su vez, cuando el contacto humano-animal es mínimo o inexistente, los animales generalmente resultan atemorizados ante la sola presencia del hombre lo que les trae aparejado respuestas de miedo y estrés (Zulkifli, 2013).

El miedo es una forma de reacción emocional ante un estímulo considerado a "prima facie" negativo para terminarlo, escapar de él o evitarlo (Gray, 1990). Este estado puede contribuir al estrés general, especialmente si la estimulación atemorizante es intensa, prolongada o ineludible (Craig y Adams, 1984). Las reacciones biológicas para hacer frente al estrés comprenden cambios en el comportamiento, el sistema neuroendocrino, sistema nervioso autónomo y sistema inmune. Ante muchos eventos estresantes, la primera defensa es la respuesta conductual (Hemsworth, 2003; Zulkifli, 2013; Martínez et al., 2016).

La importancia del contacto con los seres humanos y el establecimiento del vínculo humano-animal se ha estudiado en muchas especies de ungulados, y en todas se ha identificado que el contacto temprano y positivo da como resultado animal domesticados que muestran menos temor al hombre (Hemsworth y Coleman, 1998). Cabe destacar que dos de los eventos más comunes y potencialmente aterradores que enfrentan los animales de granja son los cambios repentinos en su entorno social o físico y su exposición a las personas (Jones, 1996; Newberry y Swanson, 2008).

La existencia de un período sensible para el establecimiento de la relación humano-animal resulta útil para el manejo de los animales de producción. Boivin et al. (1992) ha reportado que los animales presentan un período sensible al nacimiento y al destete para definir la calidad de las relaciones que establecen con los humanos. A su vez, Lyons (1989) y Miranda-de la Lama y Matiiello (2010) han informado que la experiencia en cabras del manejo positivo en etapas tempranas de la vida tiene efectos duraderos en tiempo.

En virtud que las cabras lecheras generalmente tienen un contacto cercano con los humanos a lo largo de su vida, y dado que el contacto temprano con los seres humanos puede contribuir a la habituación por parte de los animales es que el objetivo del presente trabajo fue el de evaluar cambios en parámetros fisiológicos, sanguíneos y de comportamiento en cabritos Saanen criados con sus madres respecto de aquellos criados artificialmente en las etapas pre y pos desleche, es decir en lactancia y en recría.

\section{Materiales y Métodos}

Se emplearon 20 cabritos raza Saanen. El periodo experimental fue de 9 semanas y constó de dos etapas: lactante (semana 1 - semana 5 de vida) y recría (semana 12 - semana 15 de vida). Durante la etapa lactante, 10 fueron criados con sus madres (CT: crianza tradicional) y 10 de manera artificial por medio de operarios (CA: crianza artificial). Los animales del grupo CT estuvieron desde el nacimiento y hasta los 60 días de vida alojados en el mismo corral que sus madres durante las 24 horas del día. Los animales del grupo CA fueron retirados de sus madres dentro de las primeras 12 horas de vida, calostrados artificialmente, y alojados en una guachera colectiva. A lo largo de los 60 días les fue suministrado dos veces al día leche de cabra en bateas individuales a razón de 1,5 litro/ animal/día. La misma persona siempre estuvo encargada de la crianza y alimentación de todos los animales en ensayo. Ambos grupos de animales tuvieron a disposición y desde la semana 2 de vida heno de alfalfa, maíz partido y agua. Durante el ensayo ningún cabrito presentó anomalías en la exploración clínica que pudieran dar sospecha de estar cursando alguna enfermedad, y que altere los resultados.

Luego de los 60 días de vida (pos desleche) los animales de cada uno de los tratamientos fueron derivados a un corral de recría en función al tipo de crianza al que fueran sometidos. En esta etapa (recría) los animales continuaron con una dieta ad libitum de heno de alfalfa, 250 gramos de maíz partido y agua.

Una vez por semana y a lo largo de todo el ensayo se midieron constantes fisiológicas como: frecuencia cardiaca, por auscultación en hemitórax izquierdo con fonendoscopio y un cronómetro (latidos por minuto). Temperatura rectal $\left({ }^{\circ} \mathrm{C}\right)$ con termómetro digital, frecuencia respiratoria mediante palpación torácica e inspección de movimientos respiratorios (1: eupnea - 2: taquipnea). También se midieron parámetros como ser: cuan predispuesto estaba a ser atrapado (1: predispuesto - 2: poco predispuesto) y presencia de actitud de patear al ser atrapado (1: no - 2: si). Cabe mencionar la evaluación se llevó a cabo teniendo en cuenta el comportamiento (huida -acercamiento) de los animales al ingresar el operario al corral. Previo se les tomó una muestra de $10 \mathrm{ml}$ de sangre por venopunción yugular para la realización de un leucograma.

Las muestras de sangre de los cabritos tanto de CT como los de CA, fueron colectadas con anticoagulante (EDTA) para la posterior realización de la formula leucocitaria relativa y absoluta. A través de una lectura de glóbulos blancos totales con microscopia óptica $10 x$ y $40 x$ en cámara de Neubauer (preparación de muestra de sangre con relación 1/20 de glóbulos blancos en solución de Turk). Para formula leucocitaria relativa y absoluta, se realizó conteo e identificación de los distintos perfiles de leucocitos en extendido o frotis sanguíneo, con tinción May Grunwald Giemsa microscopia óptica 100x. Todos los valores obtenidos de los leucocitos de los distintos 
animales, se expresan en número de células por milímetro cúbico.

El diseño experimental fue un diseño completamente al azar (DCA) con mediciones repetidas en el tiempo. Se consideraron los efectos de la semana, el tratamiento (tipo de crianza), y la interacción de ambos. Los datos cualitativos fueron analizados mediante variables dummy. La comparación entre medias fue evaluada a través del test de Tukey $(\alpha=0,05)$. El análisis estadístico de los datos se realizó a través del programa InfoStat versión 2017p.

\section{Resultados y discusión}

En principio, durante el ensayo ningún cabrito presentó anomalías en la exploración clínica que pudieran dar sospecha de estar cursando alguna enfermedad, y que altere los resultados.

En la Tabla 1 se presentan los resultados obtenidos para cada uno de los parámetros considerados durante la etapa lactantes mientras que en la Tabla 2 se informan los relevados durante la etapa de recría.

Tabla 1. Resultados obtenidos para cada uno de los parámetros considerados durante la etapa lactantes. Letras diferentes en fila representan diferencias significativas $(\alpha=0,05)$. Tamaño muestral $=100$.

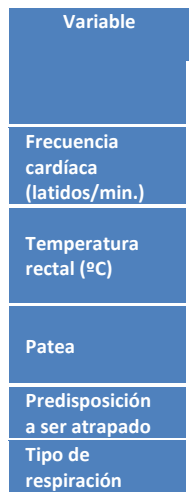

\begin{tabular}{|c|c|c|c|c|c|c|}
\hline \multicolumn{2}{|c|}{ Tratamiento } & $\begin{array}{c}\text { Error } \\
\text { están- } \\
\text { dar }\end{array}$ & \multicolumn{3}{|c|}{ Efectos } \\
\hline CT & CA & Trat. & Semana & $\begin{array}{c}\text { Trat * } \\
\text { Sem. }\end{array}$ \\
\hline 166,2 & 164,6 & 2,52 & 0,6713 & $<, 0001$ & 0,8779 \\
\hline $39,0 a$ & $38,8 b$ & 0,04 & 0,0147 & 0,0001 & 0,2708 \\
\hline $1,86 a$ & $1,56 b$ & 0,05 & 0,0001 & $<, 0001$ & 0,0028 \\
\hline $1,62 \mathrm{a}$ & $1,02 \mathrm{~b}$ & 0,02 & $<, 0001$ & $<, 0001$ & $<, 0001$ \\
\hline 1,08 & 1,06 & 0,04 & 0,7063 & 0,684 & 0,9657 \\
\hline
\end{tabular}

Las constantes fisiológicas para cabritos en cuanto a la frecuencia cardíaca es 145-240 latidos/minuto mientras que la temperatura rectal puede variar entre $37,8-39,4^{\circ} \mathrm{C}$ (Raggi y Boza, 1986). A pesar de existir una diferencia en la temperatura corporal en las distintas partes del cuerpo durante todo el día, Piccione y Refinetti (2003) han reportado una variación diaria para la temperatura rectal de $0,3{ }^{\circ} \mathrm{C}$ a $1,9^{\circ} \mathrm{C}$.

En función a los datos obtenidos en el presente ensayo en la etapa de lactantes (Tabla 1 ) no se observaron diferencias estadísticamente significativas a nivel de frecuencia cardíaca (CT: 166,20 vs CA: 164,28), mientras que si se detectaron en lo que refiere a temperatura rectal (CT: 38,99 a vs CA: $38,83 \mathrm{~b}$ ) cabe mencionar que estas diferencias se debieron al efecto de la semana de muestreo y no al tipo de crianza. A su vez durante la etapa de recría (Tabla 2) no se detectaron diferencias significativas para ninguno de los parámetros antes mencionados (Frecuencia cardíaca: CT: 174,31 vs CA: 168,56 - Temperatura rectal: CT: 38,92 vs CA: 39,02 ). Al considerar los valores promedios relevados de ambos parámetros y en las dos etapas de ensayo es posible concluir que en todas las instancias evaluadas éstos se encontraron dentro de lo considerado como normal para la especie y la edad, por lo que la presencia de los seres humanos con una frecuencia semanal en el grupo CT no alteraría los parámetros fisiológicos relativos a frecuencia cardíaca y temperatura rectal.

Tabla 2. Resultados obtenidos para cada uno de los parámetros considerados durante la etapa recría. Letras diferentes en fila representan diferencias significativas $(\alpha=0,05)$. Tamaño muestral $=80$.

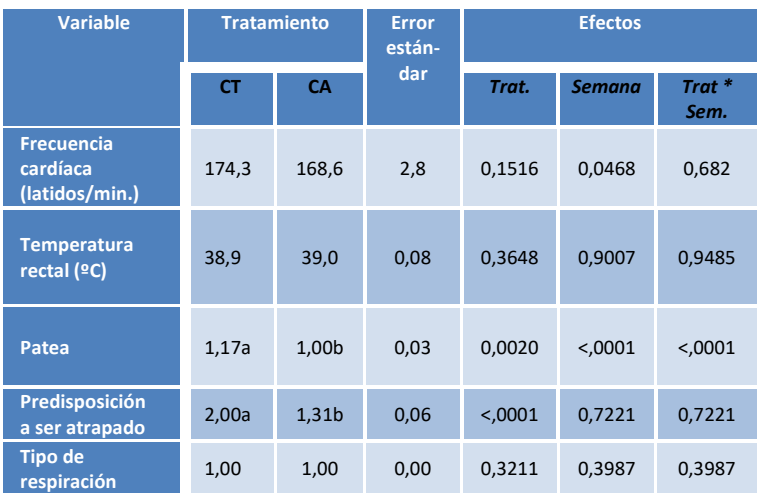

Existe una gran variedad de patrones de comportamiento que indican una reacción de miedo en los animales. La evitación, la huida y la inmovilidad son ejemplos comunes en varias especies y se pueden observar en cabras. Sin embargo, patrones de comportamiento relacionados con el miedo pueden ser contradictorios ya que tanto estrategias activas como pasivas pueden ser observadas. Es así que los animales si bien pueden ofrecer resistencia a ser atrapados, una vez logrado pueden expresar un comportamiento de evasión pasiva como la inmovilidad (Boissy, 1995; Miranda-de la Lama y Matiiello, 2010).

Al considerar las variables relativas al comportamiento tanto durante la etapa de lactancia (Tabla 1) como la de recría (Tabla2) se registraron diferencias estadísticamente significativas en lo que refiere a la predisposición a ser atrapado y la manifestación de la acción de patear al ser apresados mientras que no así en la frecuencia respiratoria.

Los resultados obtenidos en el presente ensayo coinciden con los reportado por Lyons y Price (1987) y Bergamasco et al. (2005) al trabajar con cabras adultas ya que ambos autores registraron que aquellos animales que provenían de un sistema de crianza al pie de la madre exhibían mayores distancias de fuga, es decir de evitación, y un comportamiento de huida de los humanos; por lo general eran más temerosas que las cabras criadas por humanos. A su vez Lyons y Price (1987) destacaron que estas diferencias de comportamiento no fueron acompañadas por diferencias significativas en la frecuencia cardíaca, situación similar a lo relevado en el presente ensayo en las dos etapas de evaluación. 
La acción de patear por parte del animal puede ser considerada como un mecanismo de defensa al sentirse atemorizado. Durante la etapa de lactantes (Tabla 1) si bien en ambos tratamientos se observaron animales que pateaban al ser atrapados el valor medio fue superior $(p<0,05)$ en el tratamiento $C T(C T=1,86$ vs $C A=1,56)$. Al ser considerada esta acción durante el periodo de recría si bien también se detectaron diferencias estadísticamente significativas entre tipos de crianza, cabe destacar que en el grupo CA se registraron escasos animales que patearan $(C T=1,17$ vs $C A=1,00 ; p<0,05)$. Le Neindre et al. (1996) encontraron que los animales jóvenes que eran criados junto a sus madres eran más temerosos y en situaciones particulares se comportaban de manera agresiva hacia su cuidador. En el presente ensayo y a lo largo de todo el periodo experimental los animales del tratamiento CT si bien manifestaron la acción de patear conforme se habituaron a la presencia de los operarios, básicamente por ser quienes les entregaban comida dos veces al día, menos casos de animales que pateaban al ser capturados fueron detectados (Lactante: 1,86 - Recría: 1,17). Por su parte, Lyons et al. (1988) señalaron que los factores genéticos y el ambiente postnatal temprano al que son sometidas las cabras son responsables del temperamento individual, que en gran medida persiste durante toda la vida del animal.

En la Tabla 3 es posible observar los valores relativos al leucograma de los animales a lo largo de la etapa lactantes mientras que en la Tabla 4 se presentan los relevados durante la etapa de recría.

Durante la etapa de lactantes los conteos de neutrófilos del grupo $C T$ fueron mayores $(p<0,0001)$ con respecto a los del grupo CA y los de linfocitos menores $(p<0,0001)$ durante todas las semanas, aunque no hubo diferencias en la relación neutrófilos/linfocitos. Durante el segundo período se hallaron diferencias $(p<0,0001)$ entre grupos, pero no entre semanas, aunque si la relación neutrófilos/linfocitos fue superior ( $p<0,0001$ ) en un $50 \%$ en los CT.

Se sabe que las concentraciones de algunos constituyentes sanguíneos indicadores de estrés aumentan a medida que se incrementa el grado de disconformidad, temor y defensa del individuo. Los estados de estrés pueden ser medidos a través de variables sanguíneas como el cortisol, catecolaminas, factor liberador de corticotropina, glucosa, $\beta$ hidroxibutirato, creatinfosfoquinasa y lactato al correlacionarlos con variables fisiológicas como temperatura corporal, frecuencia cardiaca, respiratoria, hematocrito y relación neutrófilos/linfocitos (Caballero y Sumano 1993; Tadich et al., 2000).
El aumento de los glucocorticoides debido al estrés produce un aumento en la liberación de neutrófilos maduros desde la médula ósea (desvío hacia la derecha de la curva de Arneth), disminuyendo su paso desde la sangre a los tejidos (Álvarez, 2002). Con cuadros de estrés crónico hay leucocitosis mediada principalmente por una neutrofilia y disminución de valores de linfocitos y eosinófilos, los cuales persisten mientras los glucocorticoides permanezcan elevados (Yagi et al., 2004). Esto se condice con lo observado, donde en ambos períodos los caprinos del grupo CT muestran un número mayor de neutrófilos e inferior de linfocitos con una mayor relación neutrófilos/linfocitos en el segundo período.

Tabla 3. Resultados del leucograma de los animales durante la etapa de lactantes. Letras diferentes en fila representan diferencias significativas $(\alpha=0,05)$. Tamaño muestral= 100 .

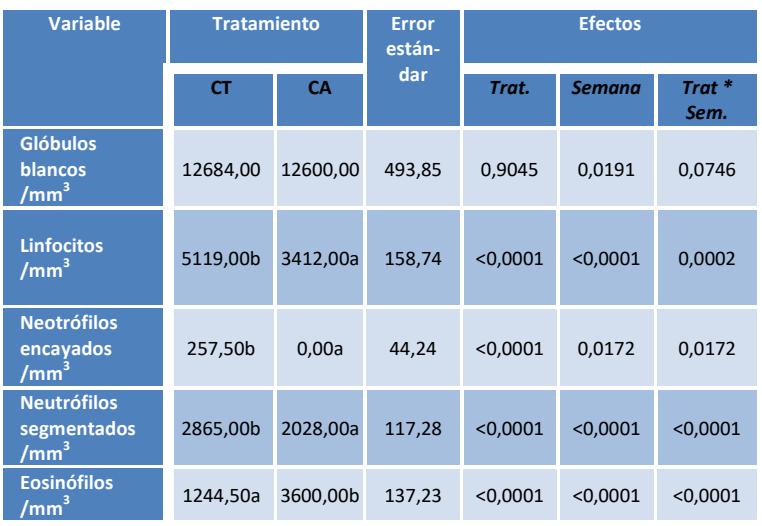

Tabla 4. Resultados del leucograma de los animales durante la etapa de recría. Letras diferentes en fila representan diferen-cias significativas $(\alpha=0,05)$. Tamaño muestral $=80$.

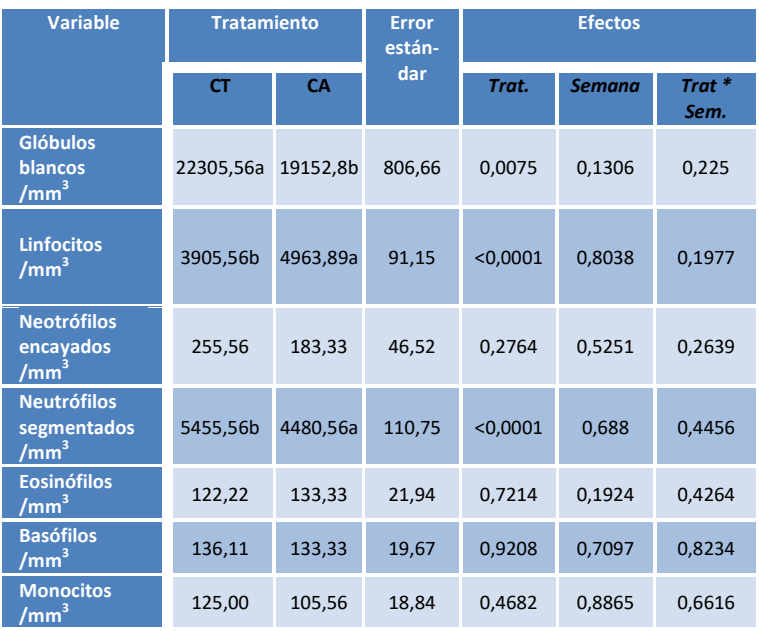

Los resultados obtenidos en el presente ensayo permiten concluir que el tipo de crianza bajo las condiciones del ensayo solo trajo a nivel fisiológico diferencias en el leucograma demostrando cierto grado de estrés cuando el animal se enfrenta al ser humano para ser sometidos a 
prácticas de manejo que ameriten su captura. Sin embargo, aquellos que han sido criados por la mano del hombre ofrecen una mayor predisposición a ser atrapado y una baja manifestación de la acción de patear al ser atrapados, tanto en la etapa de lactantes como de recría. Por lo antes mencionado se concluye que la habituación de los cabritos al ser humano en etapas tempranas incrementa el nivel de docilidad a la vez facilita la ejecución de tareas que involucren un contacto entre ambos.

\section{Bibliografía}

Álvarez E. 2002. Efecto de estímulos de diferente intensidad durante el arreo sobre algunas variables sanguíneas indicadoras de estrés en bovinos. Memoria de Título presentada como parte de los requisitos para optar al TíTULO DE MÉDICO VETERINARIO. Escuela de Medicina Veterinaria, Universidad Austral de Chile.

Bergamasco, L., Macchi, E., Facello, C., Badino, P., Odore, R., Pagliasso, S., Bellino, C., Osella, M.C., Re, G., 2005. Effects of brief maternal separation in kids on neurohormanol and electroencephalographic parameters. Appl. Anim. Behav. Sci. 93: 39-52.

Breuer K, Hemsworth PH, Barnetta JL, MatthewsC LR, Colemand GJ. 2000. Behavioural response to humans and the productivity of commercial dairy cows. Appl. Anim. Behav. Sci. 66:273-288.

Boissy A. 1995. Fear and fearfulness in animals. Q. Rev. Biol. 70: 165-191.

Boivin X, Le Neindre P, Chupin JM. 1992. Establishment of cattlehuman relationships. Appl. Anim. Behav. Sci., 32: 325-335.

Caballero S, Sumano H. 1993. Caracterización del estrés en bovinos. Arch. Med. Vet. 25: 15-29.

Craig JV, Adams AW. 1984. Behaviour and well-being of hens (Gallus domesticus) in alternative housing environments. World's Worlds Poult. Sci. J. 40: 221-240.

Gray JA. 1990. Brain Systems that Mediate both Emotion and Cognition. Cogn. Emot. 4: 269-288.

Hanna D, Sneddon IA, Beattie VE. 2009. The relationship between the stockperson's personality and attitudes and the productivity of dairy cows. Animal 3: 737-43.

Hemsworth PH. 2003. Human-animal interactions in livestock production. Appl. Anim. Behav. Sci. 81: 185-198.

Hemsworth PH, Coleman GJ. 1998. Human-livestock interactions: the stockperson and the productivity and welfare of intensively farmed animals. London, CAB International. 140pp

Jones RB. 1996. Fear and adaptability in poultry: insights, implications and imperatives. World's Worlds Poult. Sci. J. 52: 131-174.

Le Neindre P, Boivin X, Boissy A. 1996. Handling of extensively kept animals. Appl. Anim. Behav. Sci. 49: 73-81.
Lyons DM, Price EO. 1987. Relationships between heart rates and behaviour of goats in encounters with people Appl. Anim. Behav. Sci. 18: 363-369.

Lyons DM, Price EO, Moberg GP. 1988. Individual differences in temperament of domestic goats: consistency and change. Animal Behav. 36: 1323-1333.

Lyons DM. 1989. Individual differences in temperament of dairy goats and the inhibition of milk ejection. Appl. Anim. Behav. Sci. 22: $269-282$.

Martínez GM, Suárez VH, Ghezzi MD. 2016. Impacto de la relación humano-animal en la productividad y el bienestar animal de los rodeos lecheros. Rev. Arg. Prod. Ani. 36: 75-82.

Miranda-de la Lama GC, Matiiello S. 2010. The importance of social behaviour for goat welfare in livestock farming. Small Rum. Res. 90: 1-10.

Newberry RC, Swanson JC. 2008. Implications of breaking mother-young social bonds. Appl. Anim. Behav. Sci. 110: 3-23.

Piccione G, Refinetti R. 2003. Thermal chronobiology of domestic animals. Front. Biosci. 8: 258-264.

Raggi SL, Boza L. 1986. Constantes fisiológicas de la cabra. Monografías de Medicina Veterinaria 8 (Universidad de Chile). En línea:

https://monografiasveterinaria.uchile.cl/index.php/MMV/article Lview/4871.

Tadich N, Gallo C, Alvarado M. 2000. Efectos de 36 horas de transporte terrestre con y sin descanso sobre algunas variables sanguíneas indicadoras de estrés en bovinos. Arch. Med. Vet. 32: 171-183.

Waiblinger S, Boivinb X, Pedersenc V, Tosid MN, Janczake AM, Visserf EK, Jonesg RB. 2006. Assessing the human-animal relationship in farmed species: A critical review. Appl. Anim. Behav. Sci. 101: 185-242.

Yagi Y, Shiono H, Chikayama Y, Ohnuma A, Nakamura Y, Yayou K. 2004. Transport stress increases somatics cell counts in milk, and enhances the migration capacity of peripheral blood netrophils of dairy cows. J. Vet. Med. Sci. 66: 381-387.

Zulkifli I. 2013. Review of human-animal interactions and their impact on animal productivity and welfare. J. Anim. Sci. Biotechnol. 4:25. 